\title{
Improved proper name recall in aging after electrical stimulation of the anterior temporal lobes
}

\author{
Lars A. Ross ${ }^{1,2}{ }^{*}$, David McCoy ${ }^{1}$, H. Branch Coslett ${ }^{3}$, Ingrid R. Olson ${ }^{1}$ and David A. Wolk ${ }^{3,4}$ \\ ${ }^{1}$ Olson Laboratory, Department of Psychology, Temple University, Philadelphia, PA, USA \\ ${ }^{2}$ Department of Pediatrics, Albert Einstein College of Medicine, New York, NY, USA \\ ${ }^{3}$ Department of Neurology, University of Pennsylvania, Philadelphia, PA, USA \\ ${ }^{4}$ Department of Neurology, Penn Memory Center, University of Pennsylvania, Philadelphia, PA, USA
}

Edited by:

Hari S. Sharma, Uppsala University, Sweden

\section{Reviewed by:}

Russell J. Andrews, Neurological Surgeon, Private Practice, USA Gregory F. Oxenkrug, Tufts University, USA

\section{*Correspondence:}

Lars A. Ross, Department of

Pediatrics, Albert Einstein College of Medicine, 1225 Morris Park Avenue,

Bronx, NY 10461, USA.

e-mail: larsross21@gmail.com
Evidence from neuroimaging and neuropsychology suggests that portions of the anterior temporal lobes (ATLs) play a critical role in proper name retrieval. We previously found that anodal transcranial direct current stimulation (tDCS) to the ATLs improved retrieval of proper names in young adults (Ross et al., 2010). Here we extend that finding to older adults who tend to experience greater proper-naming deficits than young adults. The task was to look at pictures of famous faces or landmarks and verbally recall the associated proper name. Our results show a numerical improvement in face naming after left or right ATL stimulation, but a statistically significant effect only after left-lateralized stimulation. The magnitude of the enhancing effect was similar in older and younger adults but the lateralization of the effect differed depending on age. The implications of these findings for the use of tDCS as tool for rehabilitation of age-related loss of name recall are discussed.

Keywords: memory, temporal poles, brain stimulation, tDCS, person identity, semantic, social cognition, electrophysiology

\section{INTRODUCTION}

An inability to remember proper names is one of the most common complaints of older adults (Leirer et al., 1990). All forms of associative memory appear to decline with healthy aging (Howard et al., 2006; Yonelinas et al., 2007), but the arbitrary nature of the relationship between faces and names makes this a particularly demanding cognitive task for older adults. While there remains considerable debate regarding the neural mechanisms which drive these and other age-associated declines in cognitive function (Buckner, 2004), it has been proposed that weakening of cortical connectivity may be an important contributor (MacKay and Burke, 1990; James, 2006). In light of the rapid growth of the elderly segment of the population in developed countries around the world, there has been increased interest in interventions for optimizing and remediating cognitive aging (Daffner, 2010).

In the present study we investigated whether stimulation of the anterior temporal lobes (ATLs) by means of transcranial direct current stimulation (tDCS) to the scalp is effective in modulating the recall of proper names for well-known people (faces) and landmarks (places) in older adults. tDCS is a technique by which small electric currents are applied to the scalp. These small currents transiently modify resting membrane potentials and thereby affect the likelihood of neuronal discharge. Early animal work has revealed that the polarity of the electrodes results in a depolarization (anodal) or hyperpolarization (cathodal) of membrane potentials (Nitsche and Paulus, 2000). Applied to the human scalp, this increase or decrease of cortical excitability has been shown to extend beyond the time of the actual application of the current and may last as long as $90 \mathrm{~min}$ (Nitsche et al., 2003; Iyer et al., 2005) These longer-lasting effects may be mediated, in part, by changes at NMDA receptors, known to subserve cortical plasticity involved in learning and memory (e.g., Morris et al., 1986; Fertonani et al., 2010). Being non-invasive, safe and inexpensive, tDCS has increasingly been recognized as having great potential for neurorehabilitation and has been applied to neurotypical subjects, as well as subjects with a variety of brain pathologies (Fregni et al., 2005, 2006, 2007) including Alzheimer's Disease (Ferrucci et al., 2008). Of special interest here is that tDCS has been shown to affect language functions manifested by enhanced language learning (Floel et al., 2008; de Vries et al., 2010; Liuzzi et al., 2010) and object naming (Fertonani et al., 2010), and has already shown to be a promising tool for the rehabilitation of language function in aphasia (Monti et al., 2008; Fiori et al., 2010; Fridriksson et al., 2011).

In a recent study conducted in a young adult population, we showed that anodal tDCS can enhance proper name recall for famous faces when applied over the ATLs, particularly in the right hemisphere (Ross et al., 2010). We found these effects specifically in situations in which the recall of the name of a well-known face was not easily achieved, suggesting that the modulatory effect of tDCS on memory retrieval tasks may be particularly pronounced in cases when memory access is difficult. We attributed this enhancing effect to the modulation of neural connections that represent biographical information, a processing step that is believed to underlie person recognition. We hypothesized that this effect may be enhanced in situations in which the access to such semantic content is decreased due to weakened cortical connectivity.

In the present study we extended this paradigm to healthy older participants by presenting them with pictures of the faces of famous people and landmarks/places. The task was to recall their 
names while receiving anodal tDCS over the ATLs. The inclusion of famous places allowed us to assess the selectivity/generality of any observed effects. As with our study in young adults, we expected anodal tDCS over the ATLs to increase proper name recall for people and possibly places in cases when the name is known, but cannot be immediately recalled. In particular, tDCS would be most likely to modulate performance when the item is known, but recall of the name is slower, similar to a "tip of the tongue" state. In these instances more exhaustive memory search procedures may be required to activate name representations. This access may benefit from an decrease in resting membrane potentials and a resulting increase in the probability of neuronal discharge.

We expected to find effects similar to the ones reported in our previous study in young adults (Ross et al., 2010), but hypothesized that the modulation of recall performance may be even more enhanced in older adults due to the frequently reported normal decline in name recall in aging (James, 2006; James et al., 2008), including the significant increase in experience of "tip of the tongue" events relative to young adults (Evrard, 2002).

\section{MATERIALS AND METHODS PARTICIPANTS}

Fourteen older (seven female) neurologically normal subjects between the ages of 55 and $69(M$ age $=65)$ served as the older adult experimental group. The young adult sample was previously reported in Ross et al. (2010). They consisted of 15 (11 female) neurologically normal participants between the ages of 19 and 37 ( $M$ age $=26$ ). The older adult sample was recruited either through the University of Pennsylvania Penn Memory Center database or by flyer. All participants were right handed, had normal or corrected to normal vision and English as their first language. All participants were consented according to the Institutional Review Board of the University of Pennsylvania.

\section{NEUROPSYCHOLOGICAL ASSESSMENT OF OLDER ADULTS}

To ensure that our older adult sample met standard criteria for "cognitively normal" the following test battery, made up largely from the of the National Alzheimer's Coordinating Center's Uniform Data Set (UDS; Morris et al., 2006), was administered: minimental state (MMSE), Digit Span, Trails A/B (Tombaugh, 2004), Category fluency (Loonstra et al., 2001), 30-item Boston Naming Task (BNT; Kaplan et al., 1983), and a 10-item list-learning measure (Rosen et al., 1984). Semantic memory was measured with the Pyramids and Palm Trees test (Howard and Patterson, 1992). All participants scored within the normal range on these tasks (see Table A1 in Appendix; Mean scores were as follows: MMSE = 28.6 out of 30; digit span $=10.1$; Trails $A=33.9$ s; Trails $B=74.2$ s; Category Fluency $=24 ; \mathrm{BNT}=27.5$ out of 30; Pyramids and Palm Trees $=50.8$ out of 52 ). We also used performance on a subset of these measures to explore whether proper name recall was related to neuropsychological tests involving naming in general and whether observed tDCS effects were related to performance in naming tasks.

\section{STIMULI}

The stimulus sets were designed to contain age-appropriate stimulus material that was recognized and named correctly on approximately $50 \%$ of the trials in a pilot experiment. Difficult to name stimuli were employed to avoid ceiling effects. Fewer places were selected due to the relatively lower familiarity of our cohorts with landmarks. For the older adults, 130 photographs of famous people and 99 photographs of famous landmarks were selected. For the young adults, 165 photographs of famous people and 99 photographs of famous landmarks served as stimuli. Young participants were shown 88 pictures (55 faces, 33 places) in each of the three sessions totaling 264 stimuli and the sample of older adults were shown 77 pictures ( 44 faces, 33 places) for a total of $229^{1}$. The assignment of pictures to conditions was randomized for each participant.

\section{DESIGN AND PROCEDURE}

Experimental treatment - left anodal, right anodal, or sham was delivered on three separate days, in a randomized assignment resulting in a 3 (stimulation condition: left anodal, right anodal, or sham) $\times 2$ (stimulus category: faces or places) design. After the application of the electrodes the current was turned on and the first session started with a 2-min training session. This was followed by the experimental conditions, in which the stimulus categories were presented in a blocked, counterbalanced order. Each face or place stimulus was shown for $10 \mathrm{~s}$ during which time responses were collected. The task was to say the name of the person or place as quickly as possible, followed immediately by a left mouse click. If the name could not be recalled or the stimulus was completely unknown, the right mouse button was pressed. Individual trials were separated by a 2-s blank screen. Mean accuracy rates and reaction times (RTs) were calculated for each subject, stimulation condition, and stimulus category and subjected to a series of paired $t$-tests.

We expected the names of places to have lower recall rate and slower RTs. We further expected tDCS to have its greatest effect for items requiring longer recall times suggestive of decreased access efficiency. We therefore selected correct responses that took longer than $5 \mathrm{~s}$, similar to the analytic approach used in our prior young adult study (Ross et al., 2010). Percent correct performance for each subject and condition was log transformed to compensate for the asymmetry of the resulting performance distributions. Log transformed data were subjected to a series of planned statistical comparisons (paired $t$-tests) to assess the effect of right and left anodal stimulation on the name recall of faces and places. We suspected that there might be relationships between neuropsychological test performance in tasks involving naming so we computed Pearson correlation coefficients for performance in the BNT and Category Fluency and the overall performance in the sham condition (not limited to the $>5 \mathrm{~s}$ responses). In a subsequent analysis we compared the tDCS effects expressed as the log transformed odds ratios between older and younger subjects from our previous study directly with a repeated measures ANOVA (RM-ANOVA).

\section{tDCS STIMULATION}

Stimulation parameters were identical to that used in our prior proper-naming study on young adults (Ross et al., 2010).

\footnotetext{
${ }^{1}$ Older adults received fewer stimuli because the trial length was $10 \mathrm{~s}$ to accommodate age-related slowing in memory retrieval, as opposed to $7 \mathrm{~s}$ used in the younger sample. Overall duration of $\mathrm{tDCS}$ stimulation per session was limited to $15 \mathrm{~min}$.
} 
Participants received anodal stimulation over the right or left ATL or sham stimulation over the right or left ATL on one of three sessions on separate days. Anodal electrodes $(5 \mathrm{~cm} \times 7 \mathrm{~cm})$ were centered over the ATL using T3 and T4 electrode locations of the international 10-20 system (Jasper, 1958). The cathodal electrode was placed over the contralateral cheek below the cheekbone centered over the oral cavity. A Magstim Eldith 1 Channel DC Stimulator Plus was employed. In the experimental conditions, a $1.5-\mathrm{mA}$ of anodal current was applied for $15 \mathrm{~min}$ ( $15 \mathrm{~s}$ phase inphase out). In the sham condition, $1.5 \mathrm{~mA}$ current was ramped up (15s) and ramped down (15s) before the task began.

\section{RESULTS \\ PERFORMANCE OF OLDER ADULTS WITH ANODAL STIMULATION: ALL TRIALS}

Correct responses and RTs in left anodal, right anodal, and sham conditions were averaged and are reported separately for face and place stimuli (see Table 1). Mean accuracy was higher (faces: 62\%; places: $34 \%$ ) and mean RTs faster for faces than landmarks (faces: $3630 \mathrm{~ms}$; places: $4516 \mathrm{~ms}$ ). These results are very similar to the ones observed in younger adults (Ross et al., 2010). RTs were relatively long because participants had $10 \mathrm{~s}$ to respond and they were generated by the button press that followed the verbal response. tDCS stimulation did not significantly affect accuracy when all trials were included but was associated with a slowing of responses when place names were recalled.

\section{PERFORMANCE OF OLDER ADULTS WITH ANODAL STIMULATION AT LONGER RTS}

As laid out in the Section "Materials and Methods" and described in our study in young adults, we selected all correct responses with RTs longer than $5 \mathrm{~s}$ because we hypothesized that tDCS stimulation would be most relevant in situations when the name of the item is not immediately recalled (Ross et al., 2010). As seen in Figure 1 and Table 1, left ATL stimulation increased face naming accuracy from the baseline level (sham) of $29-40 \%[t(13)=-2.7$; $p=0.02]$. Twelve of the 14 participants improved their face naming performance after left anodal stimulation. Anodal stimulation of the right ATL did not significantly affect face name recall $[M=34 \% ; t(13)=-0.03 ; p=0.97]$, however it did improve the ability to recall names of places, from $21 \%$ in the sham condition to $33 \%$ during stimulation $[t(13)=-2.23 ; p=0.04]$. Left ATL stimulation did not significantly affect the recall of place names $[t(13)=-1.68 ; p=0.12]$.

\section{COMPARISON OF OLDER TO YOUNGER ADULTS}

The results described above were unexpected because we previously found that right ATL stimulation improved name recall for faces in young adults (Ross et al., 2010). The data for the young comparison group can also be found in Table 1. In the older adults, performance was numerically higher during right ATL stimulation but the effect did not reach significance. To compare the old and young adults directly, we computed the log odds ratios (the log 10 of the ratio of the test versus sham conditions) ${ }^{2}$ at long RTs.

We submitted the resulting values to a RM-ANOVA with the factors hemisphere (left, right), category (face, place), and the between-subject factor of group (young, old). The results are summarized in Table 2. There was a main effect of group $\left[F(1,27)=27.11 ; p<0.01 ;\right.$ Partial $\left.\eta^{2}=0.5\right]$, which reflected a greater overall enhancement of naming with tDCS in the older

\begin{abstract}
${ }^{2}$ The odds ratios were computed to derive the odds of a correct name retrieval in the stimulation condition given the base performance in the sham condition. The data were $\log$ transformed because the positive skew that resulted from the selection of responses after $5 \mathrm{~s}$ that tended to accumulate at the lower end of the performance distribution.
\end{abstract}

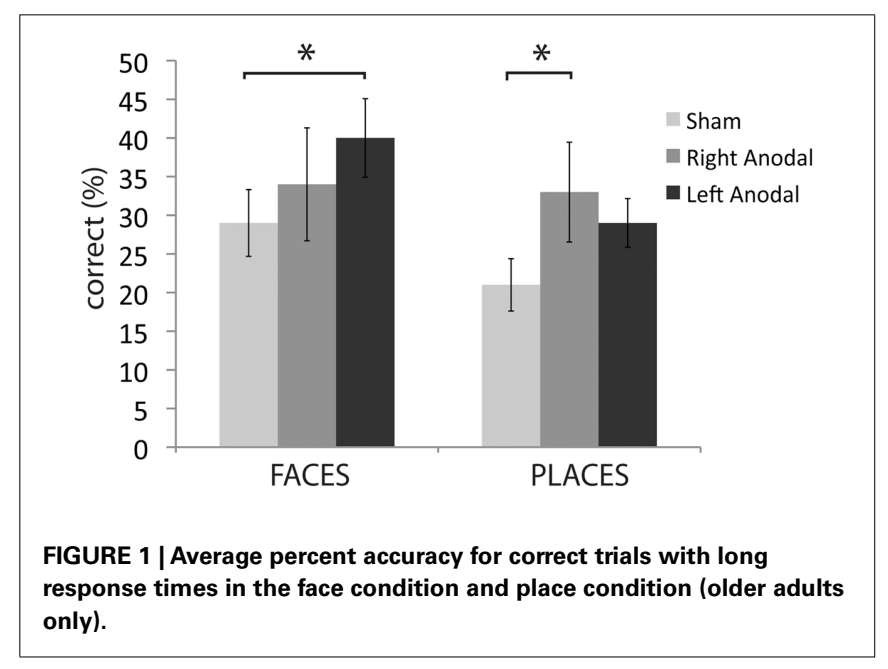

Table 1 | Accuracy (\% correct) and response times.

\begin{tabular}{|c|c|c|c|c|c|c|}
\hline & \multicolumn{3}{|c|}{ Faces } & \multicolumn{3}{|c|}{ Places } \\
\hline & SHAM & L-ATL & R-ATL & SHAM & L-ATL & R-ATL \\
\hline $\mathrm{RT}(\mathrm{ms})$ & $3649(222)$ & 3877 (229) & 3363 (157) & $4184(274)$ & $4640(458)$ & 4859 (274) \\
\hline \% old (long RT) & $29 *(4.3)$ & $40 *(5.1)$ & $34(7.3)$ & $21 *(3.4)$ & $29(3.2)$ & $33^{*}(6.5)$ \\
\hline \% young (long RT) & $27^{*}(4.8)$ & $30(7.3)$ & $38 *(3.7)$ & $32(5.6)$ & $29(5.8)$ & $21(4)$ \\
\hline
\end{tabular}

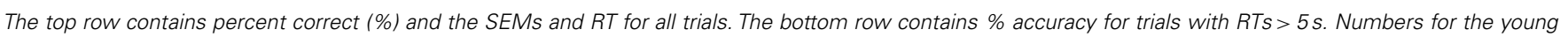

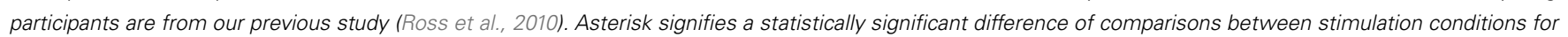
faces and places respectively. 
Table 2 | Repeated measures ANOVA table.

\begin{tabular}{|c|c|c|c|c|c|c|}
\hline Effects & $\Sigma$ of squ. & df & Mean $\Sigma$ squ. & $\boldsymbol{F}$ & $\boldsymbol{p}$ & Partial $\eta^{2}$ \\
\hline Hemisphere $\times$ group & 0.116 & 1 & 0.116 & 2.643 & 0.116 & 0.089 \\
\hline Category & 2.239 & 1 & 2.239 & 16.253 & $<0.001$ & 0.376 \\
\hline Category $\times$ group & 0.965 & 1 & 0.965 & 7.007 & 0.013 & 0.206 \\
\hline Hemisphere $\times$ category $\times$ group & 0.417 & 1 & 0.417 & 19.236 & $<0.001$ & 0.416 \\
\hline Error (hemisphere $\times$ category) & 0.585 & 27 & 0.022 & & & \\
\hline Group & 5.75 & 1 & 5.75 & 27.11 & $<0.001$ & 0.501 \\
\hline
\end{tabular}

Main effects of within-subject factors hemisphere (left, right) and category (faces, places) and the between-subjects factor group (young, old), along with interactions. $\Sigma$ of squ., sum of squares.

adults; the effect was smaller in young adults in whom face naming was selectively enhanced with right hemisphere stimulation. Further, tDCS stimulation appeared to modulate face naming to a greater extent than places as reflected by a main effect of category $\left[F(1,27)=16.25 ; p<0.01\right.$; Partial $\left.\eta^{2}=0.38\right]$. Of most interest, tDCS differentially modulated the performance of both groups, depending on the stimulation side and category, which led to a significant three-way interaction between factors of group, category, and hemisphere $\left[F(1,27)=19.23 ; p<0.01\right.$; Partial $\left.\eta^{2}=0.42\right]$.

Figure 2 illustrates this complex interaction. The left panel shows the age-dependent dissociation of hemisphere of stimulation and face naming enhancement, with older adults showing improved face naming after left ATL stimulation, younger adults after right ATL stimulation. In the right panel it is apparent that tDCS to either hemisphere disrupts place name recall in young adults whereas an improvement is observed in the aging sample. It is also worth noting that within the older adult group there is evidence of a category specific dissociation in which place naming is enhanced by right hemisphere anodal tDCS and face naming by left-hemisphere tDCS. This is evidenced by a significant interaction between stimulation side and stimulus category in the older sample $\left[F(1,13)=10.15 ; p=0.007\right.$; Partial $\left.\eta^{2}=0.44\right]$ as in the younger sample $\left[F(1,14)=10.1 ; p=0.007\right.$; Partial $\left.\eta^{2}=0.42\right]$.

\section{NEUROPSYCHOLOGICAL TEST SCORES AND NAMING PERFORMANCE}

To understand whether there was a relationship between propernaming abilities and more general semantic memory performance, we tested whether naming performance, in the absence of stimulation, was related to performance on the two neuropsychological measures involving naming: category fluency and BNT. Category fluency was strongly correlated (Pearson, twotailed) with naming accuracy in the face condition $(r=0.88$; $p<0.001)$ and approached significance in the place condition $(r=0.51 ; p=0.06)$. Similarly, performance on the BNT was strongly correlated with face naming accuracy $(r=0.7 ; p=0.004)$ and approached significance in the place condition $(r=0.49$; $p=0.07)$. These findings indicate that there is a strong relationship between face naming and more general object naming.

As mentioned in the Section "Materials and Methods" we were interested in testing whether the observed effects of electrical stimulation (stimulation - sham) were correlated with prior performance in both naming tasks but could not confirm such a relationship (Faces - BNT: $r=0.25 ; p=0.39$; Category Fluency: $r=0.02 ; p=0.03$; Places - BNT: $r=0.17 ; p=0.57$; Category Fluency: $r=0.11 ; p=0.7)$.

\section{DISCUSSION}

This study was carried out to investigate whether anodal tDCS applied over the ATLs is effective in increasing the likelihood of the recall of proper names of famous people and places in healthy older adults. In our prior study (Ross et al., 2010), we demonstrated that anodal tDCS can enhance the recall of proper names of famous individuals when applied over the right ATL. This effect was observed only in cases in which the names were not immediately available. This temporally selective effect on name recall is intuitive because tDCS is most likely to exhibit a modulatory effect when name retrieval is difficult. These stimulation effects are not likely due to a general arousal effect since we demonstrated selectivity across stimulation site and stimulus category.

We hypothesized that we would find an even larger effect of tDCS in this task in older subjects because of the known decline of proper name recall in normal aging (James, 2004; James et al., 2008). According to the transmission deficit hypothesis, normal aging is associated with a weakening of neural connections resulting in the impaired access to information with sparse connectivity in the semantic network (MacKay and Burke, 1990; James, 2006). The potential beneficial effects of tDCS would fit well with this framework as anodal stimulation decrease resting membrane potentials that in turn, could increase the likelihood of neuronal discharge in such a weakened network. Therefore, tDCS is mechanistically appealing as a rehabilitative tool for cognitive decline associated with healthy aging in addition to possible benefits in brain injured populations.

The choice of the ATLs as our stimulation site was motivated by a growing body of imaging literature suggesting this region as the location for representation of person-specific knowledge (Sergent et al., 1992; Gorno-Tempini et al., 1998; Leveroni et al., 2000; Nakamura et al., 2000; Gorno-Tempini and Price, 2001; Grabowski et al., 2001; Sugiura et al., 2001; Pourtois et al., 2005; Rotshtein et al., 2005; Ross and Olson, 2010) and neuropsychological data 

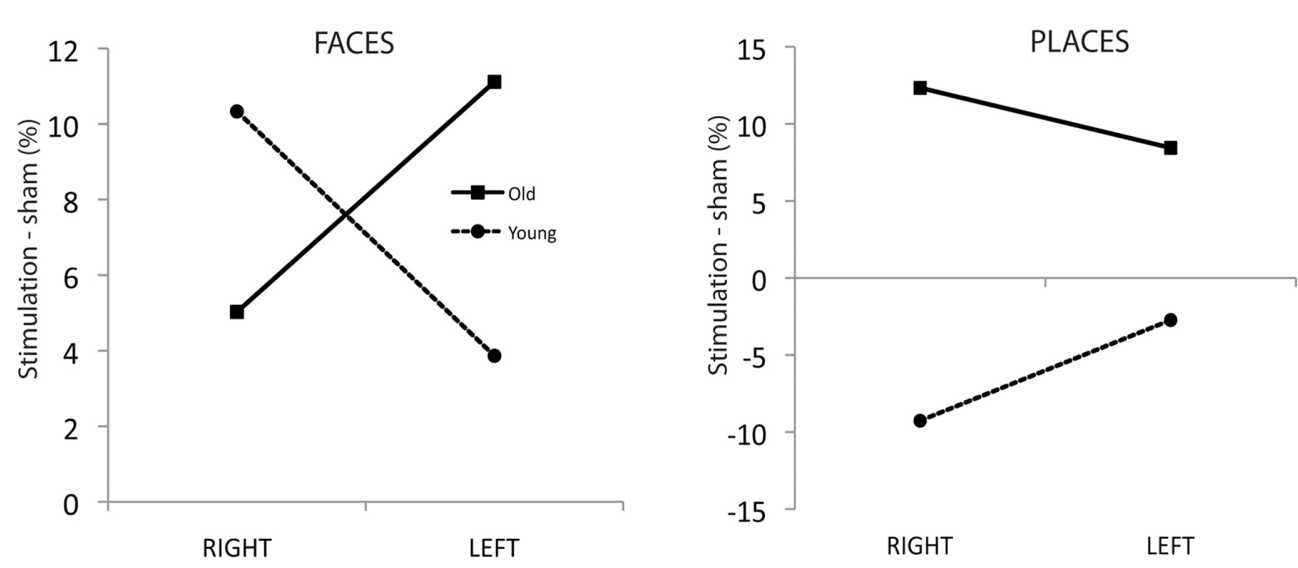

FIGURE 2 | Left panel: Face naming accuracy difference scores (\%) for ATL stimulation versus sham condition for each subject group. Right panel: Place naming accuracy difference scores (\%) for ATL stimulation versus sham condition for each subject group.

showing that focal lesions to the ATLs can cause multimodal person recognition deficits and impaired proper naming (Gainotti, 2007; Olson et al., 2007).

The observed tDCS effects on face naming in this study's older sample was surprising because it was in the opposite hemisphere to what we observed in our younger sample. Left, more so than right, hemispheric stimulation led to a marked improvement in recall of face names relative to the sham condition. However, it should be pointed out that both left and right hemispheric stimulation led to improvements in both groups. The groups differed only in the dominance of this effect. Further, unlike in the younger sample, we observed that name recall of places was positively affected by right hemisphere anodal stimulation. This finding was unexpected, however there is some precedence for it in the literature given that ATL removal can at times cause proper name deficits for people as well as places (Tranel, 2009).

We found that object naming and fluency as assessed in the BNT and Category fluency Test was related to person and landmark naming in our task. We hypothesized that beneficial effects of tDCS may be enhanced in older participants but found that the improvements in the elderly were comparable to those observed in the younger sample. Baseline language skills were not related to naming improvements during tDCS suggesting a more generalized benefit of stimulation in older adults.

It is important to note that one should be tentative in drawing inferences from tDCS effects about cortical functional organization. One reason is the limited spatial specificity of tDCS due to large electrode size and current spread such that its effects may influence a relatively wide area of potentially functionally heterogeneous cortex (Wagner et al., 2007). Another reason is that depolarization and hyperpolarization of resting membrane potentials, and therefore the enhancing and inhibitory effects, depend on the orientation of neural cell populations relative to the current flow and the direction of the current (Nitsche and Paulus, 2000). The cortical folding pattern represents an inconsistent geometry of cell orientations allowing different tDCS effects for functions that are located in close proximity to one another and is complicated by inter-subject variability in cortical folding.
While this study was explicitly designed to explore the rehabilitative utility of tDCS in age-associated naming deficits rather than to provide a detailed account of the functional specialization of the ATLs for proper name recall, we can at least speculate on possible reasons for the differences between tDCS effects in younger and older individuals, keeping the above caveats in mind. A common finding in the aging literature is evidence of functional reorganization reflected by a hemispheric asymmetry reduction in BOLD signal (Cabeza, 2002; Dolcos et al., 2002; Li et al., 2009). The hemispheric asymmetry reduction in older adults (HAROLD) model poses that these changes reflect compensatory processes due to under- or inefficient recruitment of the specialized, unilateral networks activated in young adults. This compensatory recruitment is often in homologous regions in the contralateral hemisphere from the cortical regions activated in young adults. Furthermore, such compensatory behavior may be differentially instantiated dependent on task and subject related factors (Cabeza, 2002; Park and Reuter-Lorenz, 2009). It is possible that the observed effects here reflect promotion of this compensatory activity in the left ATL with anodal tDCS over this structure in support of ineffective unilateral right ATL recruitment, which underlies face naming in young adults. Direct right ATL stimulation, which produced a more modest, but not statistically significant benefit in the older adults, may be less effective in overcoming inherent inefficiencies than stimulation of alternative networks to support performance. Such a result is not without precedent as Sole-Padulles et al. (2006) described a face-name episodic encoding task in which repetitive transcranial magnetic stimulation to the prefrontal cortex enhanced activity in this region and improved memory performance in older adults presumably by compensating for inefficient memory networks.

Another possible explanation for the observed differences is based on the different stimulus material used for young and older participants. In the young adults, knowledge of most of the famous faces was likely acquired relatively recently. In contrast, the knowledge about faces (and places) in our older sample was presumably learned decades ago (such as the face of Marilyn Monroe). The search for the name of a person that has been learned a long time ago may place different demands on the neural networks 
underlying name recall and may involve a more comprehensive search through long-term memory.

A different explanation for the observed differences between older and younger participants derives from the complexity of the processes underlying person recognition and proper naming. The access to phonological representations in a name recall task is thought to be a late step in a serial information processing hierarchy (Bruce and Young, 1986; Burton et al., 1990; Galdo Alvarez et al., 2009). In our previous publication (Ross et al., 2010) we speculated that the right-sided effects in younger adults were due to the modulation of right hemispheric neural networks underlying the recognition or retrieval of person-specific semantic information rather than the recall of its name. This idea is supported by studies of patients with focal ATL lesions that have shown that right-sided damage tends to be associated with the loss of feelings of familiarity and access to biographical information (excluding the name; Gainotti, 2007; Gainotti et al., 2010). In our experiment this enhanced ability for person recognition may in turn have affected the downstream retrieval of left-hemisphere phonological representations (Burton and Bruce, 1992). It is possible that in older adults, tDCS enhanced this later stage which has been shown to have a left hemispheric focus (Tranel et al., 1997; Grabowski et al., 2001; Tranel and Jones, 2006; Tsukiura et al., 2006). Therefore, tDCS in the elderly would seem to have a most robust effect in situations similar to a true "tip of the tongue" state. Here, a person or object is already recognized and the recall of the proper name is subjectively perceived to be imminent but cannot be accessed (Maylor, 1990; Burke et al., 1991). It should be mentioned here that this phenomenon is often experienced

\section{REFERENCES}

Bruce, V., and Young, A. (1986). Understanding face recognition. Br. J. Psychol. 77(Pt 3), 305-327.

Buckner, R. L. (2004). Memory and executive function in aging and $\mathrm{AD}$ : multiple factors that cause decline and reserve factors that compensate. Neuron 44, 195-208.

Burke, D. M., MacKay, D. G., Worthley, J. S., and Walde, E. (1991). On the tip of the tongue: what causes word finding failures in young and older adults? J. Mem. Lang. 30, 542-579.

Burton, A. M., and Bruce, V. (1992). I recognize your face but I can't remember your name: a simple explanation? Br. J. Psychol. 83(Pt 1), 45-60.

Burton, A. M., Bruce, V., and Johnston, R. A. (1990). Understanding face recognition with an interactive activation model. Br. J. Psychol. 81(Pt 3), 361-380.

Cabeza, R. (2002). Hemispheric asymmetry reduction in older adults: the HAROLD model. Psychol. Aging 17, 85-100.

Daffner, K. R. (2010). Promoting successful cognitive aging: a comprehensive review. J. Alzheimers Dis. 19, 1101-1122. de Vries, M. H., Barth, A. C., Maiworm, S., Knecht, S., Zwitserlood, P., and Floel, A. (2010). Electrical stimulation of Broca's area enhances implicit learning of an artificial grammar. J. Cogn. Neurosci. 22, 2427-2436.

Dolcos, F., Rice, H. J., and Cabeza, R. (2002). Hemispheric asymmetry and aging: right hemisphere decline or asymmetry reduction. Neurosci. Biobehav. Rev. 26, 819-825.

Evrard, M. (2002). Ageing and lexical access to common and proper names in picture naming. Brain Lang. 81, 174-179.

Ferrucci, R., Mameli, F., Guidi, I., Mrakic-Sposta, S., Vergari, M., Marceglia, S., Cogiamanian, F., Barbieri, S., Scarpini, E., and Priori, A. (2008). Transcranial direct current stimulation improves recognition memory in Alzheimer disease. Neurology 71, 493-498.

Fertonani, A., Rosini, S., Cotelli, M., Rossini, P. M., and Miniussi, C. (2010). Naming facilitation induced by transcranial direct current stimulation. Behav. Brain Res. 208, 311-318.

Fiori, V., Coccia, M., Marinelli, C. V., Vecchi, V., Bonifazi, S., Ceravolo, M.

with proper names, but is not necessarily limited to them. The significant relationship in our data between neuropsychological test performance in tasks involving object naming (BNT) and verbal fluency (Category fluency) and performance in our proper name task suggest that tDCS may be effective in language production in general. This is indeed supported by experiments investigating word and name retrieval in patients with aphasia (Monti et al., 2008; Fiori et al., 2010; Fridriksson et al., 2011) and healthy participants (Fertonani et al., 2010). The recall of proper names can be seen as a special case of name retrieval due to the unique and arbitrary nature of proper names that are often harder to access.

In conclusion, recall of proper names in older adults can be enhanced with electrical stimulation over the ATLs. Anodal tDCS improved proper naming of both famous faces and landmarks with left and right ATL stimulation, respectively. The effects of face naming observed here were of a similar magnitude to that of young adults, but only older adults demonstrated an effect on place naming, suggesting a potential more general benefit of anodal stimulation. Our data lend support to increasing evidence of the effectiveness of tDCS as a potential tool for the neurorehabilitation of cognitive function in healthy and pathological cognitive decline. However, more research is needed to clarify the exact conditions under which the applications of tDCS can be useful.

\section{ACKNOWLEDGMENTS}

We thank Lauren Mancuso for assisting, the Penn Institute on Aging for hosting this work, and pilot grant P30AG010124 to D. Wolk.

G., Provinciali, L., Tomaiuolo, F., and Marangolo, P. (2010). Transcranial direct current stimulation improves word retrieval in healthy and nonfluent aphasic subjects. J. Cogn. Neurosci. 23, 2309-2323.

Floel, A., Rosser, N., Michka, O., Knecht, S., and Breitenstein, C. (2008). Noninvasive brain stimulation improves language learning. J. Cogn. Neurosci. 20, 1415-1422.

Fregni, F., Boggio, P. S., Mansure, C. G., Wagner, T., Ferreira, M. J., Lima, M. C., Rigonatti, S. P., Marcolin, M. A., Freedman, S. D., Nitsche, M. A., and Pascual-Leone, A. (2005). Transcranial direct current stimulation of the unaffected hemisphere in stroke patients. Neuroreport 16 1551-1555.

Fregni, F., Boggio, P. S., Nitsche, M. A., Marcolin, M. A., Rignonatti, S. P., and Pascual-Leone, A. (2006). Treatment of major depression with transcranial direct current stimulation. Bipolar Disord. 8, 203-204.

Fregni, F., Freedman, S., and PascualLeone, A. (2007). Recent advances in the treatment of chronic pain with non-invasive brain stimulation techniques. Lancet Neurol. 6, 188-191.
Fridriksson, J., Richardson, J. D., Baker, J. M., and Rorden, C. (2011). Transcranial direct current stimulation improves naming reaction time in fluent aphasia: a doubleblind, sham-controlled study. Stroke 42, 819-821.

Gainotti, G. (2007). Different patterns of famous people recognition disorders in patients with right and left anterior temporal lesions: a systematic review. Neuropsychologia 45, 1591-1607.

Gainotti, G., Ferraccioli, M., and Marra, C. (2010). The relation between person identity nodes, familiarity judgment and biographical information. Evidence from two patients with right and left anterior temporal atrophy. Brain Res. 1307, 103-114.

Galdo Alvarez, S., Lindin Novo, M., and Diaz Fernandez, F. (2009). Naming faces: a multidisciplinary and integrated review. Psicothema 21, 521-527.

Gorno-Tempini, M. L., and Price, C. J. (2001). Identification of famous faces and buildings: a functional neuroimaging study of semantically unique items. Brain 124 2087-2097. 
Gorno-Tempini, M. L., Price, C. J., Josephs, O., Vandenberghe, R., Cappa, S. F., Kapur, N., and Frackowiak, R. S. J. (1998). The neural systems sustaining face and proper-name processing. Brain 121, 2103-2118.

Grabowski, T. J., Damasio, H., Tranel, D., Boles Ponto, L. L., Hichwa, R. D., and Damasio, A. R. (2001). A role for left temporal pole in the retrieval of words for unique identities. Hum. Brain Mapp. 13, 199-212.

Howard, D., and Patterson, K. (1992). The Pyramids and Palm Trees Test. Bury St. Edmunds: Thames Valley Test Company.

Howard, M. W., Bessette-Symons, B., Zhang, Y., and Hoyer, W. J. (2006). Aging selectively impairs recollection in recognition memory for pictures: evidence from modeling and receiver operating characteristic curves. Psychol. Aging 21, 96-106.

Iyer, M. B., Mattu, U., Grafman, J., Lomarev, M., Sato, S., and Wassermann, E. M. (2005). Safety and cognitive effect of frontal DC brain polarization in healthy individuals. Neurology 64, 872-875.

James, L. E. (2004). Meeting Mr. Farmer versus meeting a farmer: specific effects of aging on learning proper names. Psychol. Aging 19, 515-522.

James, L. E. (2006). Specific effects of aging on proper name retrieval: now you see them, now you don't. J. Gerontol. B Psychol. Sci. Soc. Sci. 61, P180-P183.

James, L. E., Fogler, K. A., and Tauber, S. K. (2008). Recognition memory measures yield disproportionate effects of aging on learning facename associations. Psychol. Aging 23, 657-664.

Jasper, H. H. (1958). The ten twenty system of the International Federation. Electroencephalogr. Clin. Neurophysiol. Suppl. 10, 371-375.

Kaplan, E., Goodglass, H., and Weintraub, S. (1983). Boston Naming Test. Philadelphia: Lee \& Febiger.

Leirer, V. O., Morrow, D. G., Sheikh, J. I., and Pariante, G. M. (1990). Memory skills elders want to improve. Exp. Aging Res. 16, 155-158.

Leveroni, C. L., Seidenberg, M., Mayer, A. R., Mead, L. A., Binder, J. R., and Rao, S. M. (2000). Neural systems underlying the recognition of familiar and newly learned faces. J. Neurosci. 20, 878-886.
Li, Z., Moore, A. B., Tyner, C., and $\mathrm{Hu}, \mathrm{X}$. (2009). Asymmetric connectivity reduction and its relationship to "HAROLD" in aging brain. Brain Res. 1295, 149-158.

Liuzzi, G., Freundlieb, N., Ridder, V., Hoppe, J., Heise, K., Zimerman, M., Dobel, C., Enriquez-Geppert, S., Gerloff, C., Zwitserlood, P., and Hummel, F. C. (2010). The involvement of the left motor cortex in learning of a novel action word lexicon. Curr. Biol. 20, 1745-1751.

Loonstra, A. S., Tarlow, A. R., and Sellers, A. H. (2001). COWAT metanorms across age, education, and gender. Appl. Neuropsychol. 8, 161-166.

MacKay, D. G., and Burke, D. M. (1990). "Cognition and aging: a theory of new learning and the use of old connections," in Aging and Cognition: Knowledge Organization and Utilization, ed. T. M. Hess (North Holland: Elsevier Science), 213-263.

Maylor, E. A. (1990). Recognizing and naming faces: aging, memory retrieval, and the tip of the tongue state. J. Gerontol. 45, P215-P226.

Monti, A., Cogiamanian, F., Marceglia, S., Ferrucci, R., Mameli, F., MrakicSposta, S., Vergari, M., Zago, S., and Priori, A. (2008). Improved naming after transcranial direct current stimulation in aphasia. J. Neurol. Neurosurg. Psychiatr. 79, 451-453.

Morris, J. C., Weintraub, S., Chui, H. C., Cummings, J., Decarli, C., Ferris, S., Foster, N. L., Galasko, D., Graff-Radford, N., Peskind, E. R., Beekly, D., Ramos, E. M., and Kukull, W. A. (2006). The Uniform Data Set (UDS): clinical and cognitive variables and descriptive data from Alzheimer Disease Centers. Alzheimer Dis. Assoc. Disord. 20, 210-216.

Morris, R. G., Anderson, E., Lynch, G. S., and Baudry, M. (1986). Selective impairment of learning and blockade of long-term potentiation by an N-methyl-D-aspartate receptor antagonist, AP5. Nature 319, 774-776.

Nakamura, K., Kawashima, R., Sato, N., Nakamura, A., Sugiura, M., Kato, T., Hatano, K., Ito, K., Fukuda, H., Schormann, T., and Zilles, K. (2000). Functional delineation of the human occipito-temporal areas related to face and scene processing: a PET study. Brain 123, 1903-1912.

Nitsche, M. A., Liebetanz, D., Lang, N., Antal, A., Tergau, F., and Paulus,
W. (2003). Safety criteria for transcranial direct current stimulation (tDCS) in humans. Clin. Neurophysiol. 114, 2220-2222; author reply 2-3.

Nitsche, M. A., and Paulus, W. (2000). Excitability changes induced in the humanmotor cortex by weak transcranial direct current stimulation. J. Physiol. 527, 633-639.

Olson, I. R., Plotzker, A., and Ezzyat, Y. (2007). The Enigmatic temporal pole: a review of findings on social and emotional processing. Brain 130, 1718-1731.

Park, D. C., and Reuter-Lorenz, P. (2009). The adaptive brain: aging and neurocognitive scaffolding. Annu. Rev. Psychol. 60, 173-196.

Pourtois, G., Schwartz, S., Seghier, M. L., Lazeyras, F., and Vuilleumier, P. (2005). View-independent coding of face identity in frontal and temporal cortices is modulated by familiarity: an event-related fMRI study. Neuroimage 24, 1214-1224.

Rosen, W. G., Mohs, R. C., and Davis, K. L. (1984). A new rating scale for Alzheimer's disease. Am. J. Psychiatry 141, 1356-1364.

Ross, L. A., McCoy, D., Wolk, D. A., Coslett, H. B., and Olson, I. R. (2010). Improved proper name recall by electrical stimulation of the anterior temporal lobes. Neuropsychologia 48, 3671-3674.

Ross, L. A., and Olson, I. R. (2010). Social cognition and the anterior temporal lobes. Neuroimage 15, 3452-3462.

Rotshtein, P., Henson, R. N., Treves, A., Driver, J., and Dolan, R. J. (2005). Morphing Marilyn into Maggie dissociates physical and identity face representations in the brain. Nat. Neurosci. 8, 107-113.

Sergent, J., Ohta, S., and Macdonald, B. (1992). Functional neuroanatomy of face and object processing: a positron emission tomography study. Brain 115, 15-36.

Sole-Padulles, C., Bartres-Faz, D. Junque, C., Clemente, I. C. Molinuevo, J. L., Bargallo, N., Sanchez-Aldeguer, J., Bosch, B., Falcon, C., and Valls-Sole, J. (2006). Repetitive transcranial magnetic stimulation effects on brain function and cognition among elders with memory dysfunction. A randomized sham-controlled study. Cereb. Cortex 16, 1487-1493.

Sugiura, M., Kawashima, R., Nakamura, K., Sato, N., Nakamura, A., Kato, T. Hatano, K., Schormann, T., Zilles,
K., Sato, K., Ito, K., and Fukuda, H. (2001). Activation reduction in anterior temporal cortices during repeated recognition of faces of personal acquaintances. Neuroimage 13, 877-890.

Tombaugh, T. N. (2004). Trail making test $\mathrm{A}$ and $\mathrm{B}$ : normative data stratified by age and education. Arch. Clin. Neuropsychol. 19, 203-214.

Tranel, D. (2009). The left temporal pole is important for retrieving words for unique concrete entities. Aphasiology 23,867 .

Tranel, D., Damasio, H., and Damasio, A. R. (1997). A neural basis for the retrieval of conceptual knowledge. Neuropsychologia 35, 1319-1327.

Tranel, D., and Jones, R. D. (2006). Knowing "what" and knowing "when". J. Clin. Exp. Neuropsychol. 28, 43-66.

Tsukiura, T., Mochizuki-Kawai, H., and Fujii, T. (2006). Dissociable roles of the bilateral anterior temporal lobe in face-name associations: an eventrelated fMRI study. Neuroimage 30, 617-626.

Wagner, T., Valero-Cabre, A., and Pascual-Leone, A. (2007). Noninvasive human brain stimulation. Annu. Rev. Biomed. Eng. 9, 527-565.

Yonelinas, A. P., Widaman, K., Mungas, D., Reed, B., Weiner, M. W., and Chui, H. C. (2007). Memory in the aging brain: doubly dissociating the contribution of the hippocampus and entorhinal cortex. Hippocampus 17, 1134-1140.

Conflict of Interest Statement: The authors declare that the research was conducted in the absence of any commercial or financial relationships that could be construed as a potential conflict of interest.

Received: 29 June 2011; accepted: 27 September 2011; published online: 12 October 2011.

Citation: Ross LA, McCoy D, Coslett HB, Olson IR and Wolk DA (2011) Improved proper name recall in aging after electrical stimulation of the anterior temporal lobes. Front. Ag. Neurosci 3:16. doi: 10.3389/fnagi.2011.00016

Copyright ( $(2) 2011$ Ross, McCoy, Coslett, Olson and Wolk. This is an open-access article subject to a non-exclusive license between the authors and Frontiers Media $S A$, which permits use, distribution and reproduction in other forums, provided the original authors and source are credited and other Frontiers conditions are complied with. 


\section{APPENDIX}

Table A1 | Neuropsychological test performance.

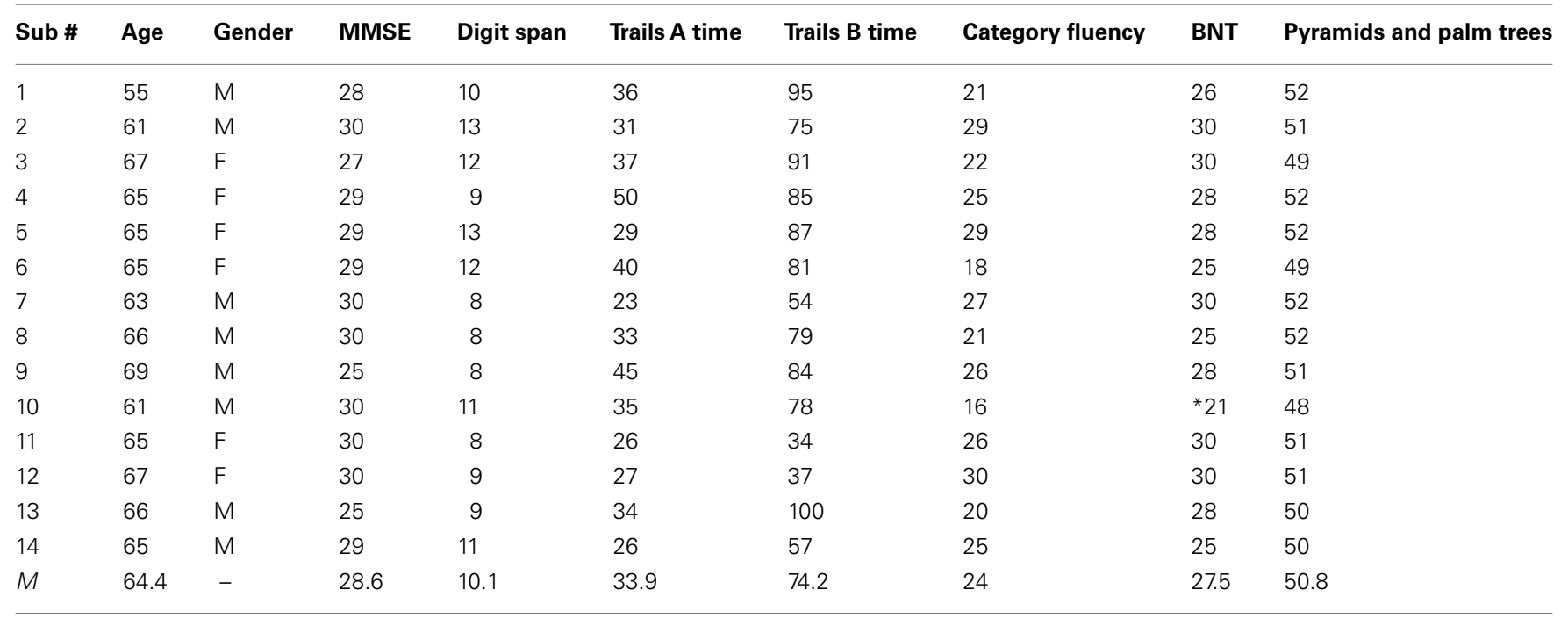

Individual and mean scores on neuropsychological tests administered to older participants. The MMSE score is out of 30. Digit span was calculated by forward total score (total $=16)$. The BNT score represents total correct (number correct without a cue + number correct with semantic cue; phonetic cues not included) and is out of 30. The pyramids and palm tree score is out of 52. The one abnormal score is marked by an "*." 